\title{
FEDERATION SEISMOLOGIQUE EUROPEENNE
}

\author{
(F. S. E.)
}

\section{COMPTES-RENDUS DES SEANCES DU CONGRES REUNI \\ A VERONE DU 13 AU 15 SEPTEMBRE 1950}

\author{
Rediges par le Secrétaire da Congres: E. Peterscunutro \\ aver une introduction de M.lle I. LEmManN.
}

Publiés par le Secrélaire P. Caloo.

\section{INTROIIUCTION}

Au mois de juillet $19+7$ l'Association Internationale de Sóismolowie a tenu a Strashourar une rémion qui devait reprendre la collahoration internationale interrompue par la gruerre. Les participants ont iprouve un tel sentiment d'entichissement par les contacts entre colligues que l'éventualite de rémions plus fréquentes de ce renre a clé disculce. Au cours de l'Assemblée Génerale de l'Lnion Géodesique el Geoplysique internationale tenue a Oslo en 1948 , l'idés de la constitution d'une société séismologrique européenne a été exprimie. Tous les seirmologues europiens et non pas seulement les délé„wás envoyes aux rémions de l'U.G.G.l. devaient en faire partie. Ce projet a dé discule el au rours d'une réception a Akerslus, on s'est mis d'accord pour fonder une telle société.

Ine lettre circulaire diflusée an mois de mai $19-19$ a rendu compte des idees formant la lase de celle initiative:

Beauconp de séismologues européens travaillent dans un isolement plus ou moins grand. On est d'avis que les recherches séismolowiques seraient encouratées et avanceraient mieux si l'on pouvait creer entre eux un contact meilleur el élablir une collahoration pour le solution de prohlemes spéciaux.

Au déhut du siecle l'Europe dépassait de loin les Etats-Lnis dans le domaine séismologrique. Actuellement cet élat de choses est completement change, non seulement en raison des moyens financiers plus importans mis a la disposition des chercheurs mais aussi par suit? de la collahoration ćtendue existant. rollahoration dont limportance est romprise par tous. Dans differents domaines des résultats tres remarqualiles ont éte arquis - dans un temps éconnament court frate à la collaboralion élahlie sur une large échelle.

Ides reismologrues europiens ne se réunissent que tous les trois ans a l'occasion des reunions de l'A.I.S. Celle-ci est une organisation mondiale: l'orde de jour est charare de sorte que le tempss qu'on peut consacrer aux discussions des différents problimes est limite el qüil est imposible d'examiner des questions spécififuement europrennes. Beatroup de seismologues europrens ressentent l'alsence d'une 
société eurcpéenne analogue à la Societe Séismologrique Américaine, une sociéte qui par des réunions plus frépuentes d'un nombre plus élevé de éismologues, permellatil la diseussion des problemes et provotuerait une meilleure collaboration.

Les séismolognes etroprens ont éce invites à former une telle sociéli.

Pour des ratsons de commodite pratipue, les invilations ont ele diflusécs par le Bureau luternalional de Séismologie de Suasboury qui a éralement aceepte de recevoir les reponser.

25 seismologues de 13 pays dillérents répondirent. Tous se moutrirent favorables à la proposition el quelpues uns exprimient leur grand interel. 11 fut decide de chereher à oraniser une réumion constitulive.

Des difficulies plus importantes quion pouratit le prevoir sont apparues. Afun d'éviter des frais de deplacement tris mrand el de simplifier les formalités de passeport, il a parn nécesaire de tenir celte réunion dans un pays situe de facon centrale en fiumope. Quelgues propositions s'étant réceles impratiquables, l'lstituto Nazionale di Geofisiea de Rome sous l'impulsion des Prolesseurs Medi, Directeur el Caloi, géphysicien en chef, a accepte d'orwaniser la rémion constitutive dans une ville de l'ltalie septentrionale. le choix s'est porte sur la ville de Vérone où elle a eu lieu du 12 au 16 septembre 1950.

L'Istituto Nazionale di Geofisica a montre une hospitalite unique, tous les participants élant ses holes pendant les quatre jours qu'ils ont passé à Verone.

Au mois de décembre 1949 une proposition ale "by-laws" blahores par R. Stoncley el I. Lehumam (voir pane 601) fut dillusoe. Des commentaires qui l'aecompagnaient, indiguaient dilferents points de vue sur la constitution de la societé on organisation comme olle a été dénommée, points de vue exprimés dans les réponses regues aux invilations.

On a proposé de constituer une sociéte seismologique "libre" où lous les séismologues poumaient être atmis el qui serait indépendante tes organisations existantes.

Dans leurs réponses, quelques uns des séismologues adhéraicul fortement à cette idée tandis que d'autres pensaient quil fallait établir un rapport avee l'A.I.S. et l'L.G.G.l.: sinon il serait impossible d'ohtenir des subventions aux frais d'orwanisation ot de deplacement. Certains enfin ont propose la constitution d'une commission dependant de l'A.I.S.

Avant l'ouverture de la réunion de Verone. l'lstituto Nazionale di Geofisica, a lait distribuer un projet de statuts (roir page 603). Ce projet de même que l'ordre du jour (voir pare 581 ) ont mis atu premier plan la question d'un rapport éventuel aree l'A.l.s.

\section{TII!)}




\section{ORDRE DL JOUR} Talien.

1". Ouverture du Congres par un representant du Gouvernement

2". Nomination du Bureau du Congress.

$3^{\circ}$. Dicussion sur le but d'une organisation sópsmologique européenne et sur ses relations avec l'A.I.S.

1.". Etablissement et discussion des statuts.

5". Presentations et discussions des communications seientifiques $(*)$ :

"Polarization of the S-phatese (R. SToxeley).

"Lat constitution de l'Athantiquen (J.P. Rotsis:

"Intersuchungen ïber Mikroseismik wahrend des Krieses in Doutichland n (E. Handerwigi.

"Les ratacléristiques a domes aux séismographes electromagnéti-

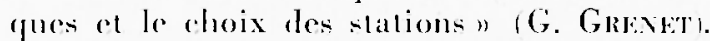

"Acoplamiento al mismo galvanómetro de dos aparatos zenitales de diverso periodon ir. Gowaz Guillawovi.

"A porlable electromagnetic seismograph" (P.I. WILLMone).

"Le nouveau reglement du Service Seismologique en lougoslavie, en usage a partir du l. janvier 1949" (J. Murafovicl.

"Sulla superfiede di discontinuila alla profondita di $950 \mathrm{~km}$ ea $"$

iP. Carom, F. Penovacio.

6". Eilaction du Conseil de la Faderation.

\section{HORARE DES SEA NCES \\ Verrede 1:) Septembre 19.30}

9 hemes: Ouverlure du Congres par Monsier A. Fedeli, Manie de la ville de ferome of premiere seance de travail.

15 heures: Seance de la Commiseion Restreinte.

17 heures 30: Siance de travail.

\section{Jeudi 14 Septembre 7950}

9 hemes: Sianer de tranail.

16 heores 30: Séance de lit Commi-cion Resireinte.

17 heures 30: Siance de travail.

\section{Venciredi 15 September 1950}

8 heures: Excursion geologique an Lac de Garle: cturle de l'amphithêtere morainigue de Peschiera, zone seismigue de hordure.

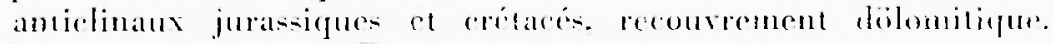
liepals en commun à Riva.

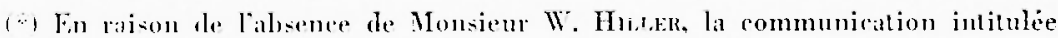

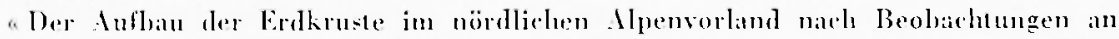

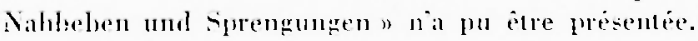


20 heures 30: Banquet offert par le Conscil municipal de Verone sous la présidenee de Mlonsienr le Maire.

\section{LISTE DES PARTICIPANTS}

Albmagne: Dr. E. Hardowign, Friedrielestr., 17 - Mänchen 13; Dr. II. Menzel, Geophysikalisches Instilut der Lnisersitait, Rothenbaumchaussec, 33 - Ilamburg; Prof. L. Mintrop, Barkhovenallee 36 Essen-II erden; Dolt. G. A. Selutze, Herzberger-Landstrasse 180 - Göllingen.

Daxbank: Nlle l. Lehmann, Geodaclisk Institul, Seimological Department, Provianlatalen. Kobenharn.

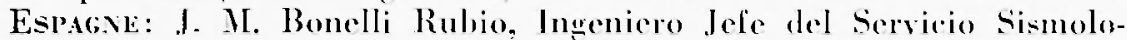

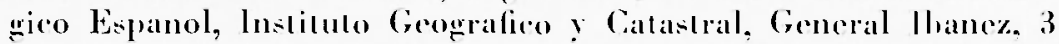
- Madria; F. Gomez-Guillamon y Guillamon, Ingeniero Jele del Observalorio Sismologieo de Malaga.

Fravee: Dr. G. Grenel, Directeur de l'Institut de Méliorologie, Université - Alger; Ing. Fi. Peterselomitt. Institul de Physigue du Glohe, 38, Boulevard at'Anvers - Strasbourgr Prof. J. P. Rothe. Directeur de l'Instiut de Physigue du Gloles, 38. Boulevard d'Anvers - Strasbourg.

Gravof-Brerage: Mr. E. F. Baxter, Fichlhouse Tertace, Durham; Prof. R. Stoneley. I niversily of Cambridge. Departument of fieodesy and Geophysies, Downing Place - Cambridge: Dr. I. I. Wilmore, Universily of Cambridge. Department of Geode-y and Geophysies, Downinᄈ Place - Cambridge.

Irabe: Mile Prof. G. Aliverti, Istituto Superiore Navale. Napoli: Prof. Pierro Caloi, Geofisieo Capo Istimen Naz. di Geofisica, Citla Universilaria, Roma. Prof. M. Giorqu. Istilulo Naz. di Cioufisica, Roma: Prol. G. Imbò, Direllore Osservalorio V esuliano. Napoli; On. Prof. Enrieo Medi. Dipettore Istitulo Naz. di Ceofisical, Roma: Prof. Carlo Morelli. Osservatorio Geofisico, Trieste: Dr. Mario De Panfilis, Istilulo Naz. di Geofisica. Roma: Dr. Guido Pannocehia, Osservatorio Geofisico. Patia: Dr. Franceseo Pr.ronaci, Istitulo Naz. Geofisica, Roma: Dr. Paolo Enilio Valla. Istilulo Naz. Geofisial, Roma.

Susse: Dr. Edmond Guyo!, Directemr de l'Ohervatoire Cambonal Neuchatel: Mr. Pierre Mereier, Eniversité, Laboraloire de Giophysique - Lausamne.

\section{COMIPTE-RENDUIS DES SEANCES \\ Premiere secuce, mercredi l2 septembre (matin)}

Toules les séances de travail ont eu lieu dans la salle de réunion du Muséum d'Histoire Naturelle de Vérone. Monsieur Fedeli, Maire de la ville pononce une courle allocution de bienvenue. Ie? 
Professcur Medi accueille les participants au nom de l'Istiluto Nazionale di Geofisica. Il soulaite en particulier que de même qu'un séisme peut toucher tout pays, il se lorme une séismicité des coeurs sans distinction de nationalité. Le Professeur Stoncley F.R.S. président de l'A.I.S., remercie le Gouvernement Italien, le Conseil Municipal de Vérone el l'Istitulo Nazionale di Geofisica de l'accueil réservé aux participants.

Après le départ des autorités ofliciclles les congressistes procèdent par acclantion a l'election du huraau.

Sont ilus:

Prisident: Monsieur Medi;

Vice-Président: Mademoiselle Lehmamn:

secretaire: Monsieur Petersehmitt.

Le programme de la séance de travail est introduit par le président Medi qui rappelle l'ordre du jour. Au moment de la constitu. tion de celle organisation séismologippe curopéenne, il lui paraît opportun d'engager la discussion sur des bases tres larges afun d'établir les principes generaux de lorganisation.

A la demande du préxident Stoneley, le Professeur Rothe Sécré. taire Gencial de l'A.J.S. fait un expose des principes qui regissent cel organisme el en déduit les relations qui pourront exister entro l'A.I.S. al l'organisation séismologique européenne. L'A.I.S. est l'une des sepl Asociations constituant l'Union Géodésique el Géoplysique International des Unions seientifiques. L’A.I.S. n'a done qu'une autonomic restreinte: en particulier elle ne possede pas de ressources financières autonomes et elle ne convoque pas les assemblées générales, ces deux points élant du ressort du bureau de l'U.G.G.l. Par ailleurs ce ne sont pas des personnes physiques qui sont membres de l'U.(i.G.l. mais des pays. Claque pays nembre peut envoyer des dé. legues aux assomblées éénérales. Les différentes nations du erlobe se répartisent actuellement en trois ratégories:

a) Les nations membres litulaires. Les délégués ont droil de vote dans toutes les questions: statulaires, hudgétaires el scientifiques:

b) Les nations invités: celles-ci peuvent envoyer des délégués qui nont pas droit de rote nais peurent presenter des communications scientifiques.

c) Les nations qui ne peusent encore faire partie de l’U.G.G.I. a savoir les deux Republiques Allemandes. la Chine el le Japon.

Monsieur Rothé s'excuse aupres des participants allemands d'avoir l'obliwation de rappeler ces faits, mais il estime qu'il faut aborder les questions avec entière franchise. Lu 12 septembre 1950 l'état de fucre subsiste toujours entre l'Allemagne el les Alliés, mais il est probable que cette situation se modifiera dans un avenir prochain. Les allemands de l'ouest sont d'ailleurs déjà représentés au Conseil de l'Europe it Strashourar.

De cel état de fait on peut déduire une conclusion trés impor- 
lante: dans foutes les discussions qui ront avoir lien il ne faul pats oublier qu'en ee qui concerne les rapports à ébablir entre l'oramisa. lion europécnue el l'A.l.S., il existe des organismes directeurs qu'il est nexessate de ronsulter el dont il faut avoir l'approbation.

Le president Stoneley met en lumiere les diffieultés d'une orannisation qui serait uniquement place dans le eadre de l'l.C.C.l. Dans ce calle en effet une remion libe de memberes ne pouratit aroir lieu.

Le président Medi pense qüil serait possihle de ereer une rénion non de pays mais de personnes sans distinction de nationalite.

Le Prolesseur Rothe indique les deux points de vue extrêmes. D'une part on pourail envisager un organisme analogue a la Commission Geolesifue Europénne. Cetle rommission serait une soussection de l'A.I.S. Des réunions plus frequentes a caracteres alsolu. ment uftects pouraticnt avoir lien. D'autre part, il y aurait l'hypothèse d'une société eompletement privé analogue a la Sociéte Séimologique Amerieane. Fntre res deux positions extremes il y aurait peutêtre lieu le trouver une solution intermédiaire. Fn tous les cas il ne faulrate pas oublier le point de vue des indiateurs de l'iles de rencontres phus frépuentes, Mestemoiselles lehmann et Bohr, Monsieur Clatrier, rencontres qui devaient permettre la discossion de problimes seientifiques et surtout pratiques ou les seismolowues s'en. lr'aideraient dans l'élude de questions particulieres. En eflet aux ansemblés generales de l'U.G.G.l. le programme es trop chargé pour quion puisse trater les questions aved tout le temps nécessaire.

Le Professeur Mintrop insiste sur le fait que les problimes de. cette organisation et ceux de l'A.I.S. sont différents. Il s'anit arant tout l'étulier des problemes europeens el ceei peut se faire surloul par l'élude des séismes rapprochés qui, en Europe, ne sonl généralement pas assez intenses pour être enregistrés à mrande distance.

La discussion qui suit met en lumiere les arantignes el inconve. nients des différents as possibles.

\section{I". Commission dépendant de l'V.C.G.I.}

a) feantages: étant domne le caraetione offeciel llune telle commission on serail assure l'avoir l'appui des divers pouremements en particulier pour l'obtention des moyens finaneiers necessiares à l'organisation des rémonions et au remboursement des fratis de deplacements. IIM. Stoneley. Rothei. En tous eas les londs de l'U.ficil. peuvent uniquement etre employes an fonctionmement des sicretatiats et des serviees des differentes associations I.S.S. par exemples a la publication des comples-rendus de seances el des mémoires seientifipues.

b) Inconténients: une rommission qui serait une sous-section de l'I.S.A. devait pour etre reconnue applieguer les slatuts de l'I.G. G.I. A. Stoneley. En particulier la participation alevait etre fondé sur les pays membres, ee qui excherait actuellement la presence des savants allemands. Pour realiser la participation de ces derniers, il 
serait posilate de convorguer un eollogue ou un symposium sur un problime délerminé, colloque pour lequel l'LNESCO pourrait débloquer des londs; dans ae cats le nomber de participants devait être limile (II. Rothe et il est pobbrble que le gomsermement allemand refuseratit a ses ressortissants de participer a un collogue seulement 1.I. Hardlwipl.

Enfur les membres d'une commisson sarent délegués par leur Gouvernements respectifs ce qui limiterait leur nombre. Les seismologues europecoss ne pouratient en latre partic de leur plein gre.

\section{2". Sociele privere.}

a) feantrgess: on anrait liberte romplete pour l'admission des member el on pourrait travaller de facon alsolument independante.

h) Incomernionts: il serat diflicile d'olstenir des appuis offciels. Plusieurs participants font resortir que les conditions semi-

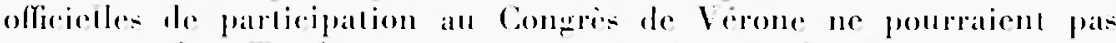
se renouveler. T'andis que pour l'assemble de Strasbourg on pour

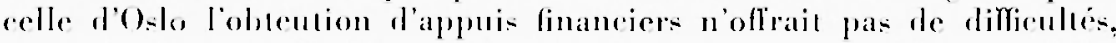
pour le Congres de Verone la siluatione etate trie different MII. Stoneley, Bonelli, Rolher.

Avant dinterrompre les débats le président Medi degage l'opinion gencrale de l'S-semblée, resta-die ereer une organisation qui ail des rapports d'ume parl suffisamment etroits aver l'I.S.A. el l'L

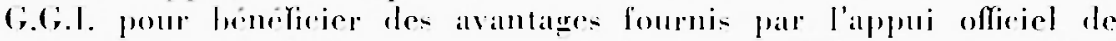

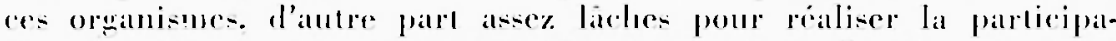
tion du plus grand nombere posiblole de membres. Lal séance est interompue a ll bemes 45.

1 la reprise de la séance 12 lemes 20 le president Medi dégage les resulats des exbanges d'ides qui ont cu lien pendant l'intermption. Il parail opportun de constituer une orwani-ation seismologique europernne er d'en etablir les statuts. Ces staluls seraient provisoires ot valables un an. A lassomblio de Bruxelles ils seraient sommis à l'avis. du Conseil de l't.C.ci.l. Parallelement ou a la suite de l'as-

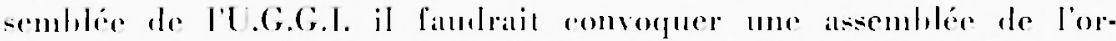

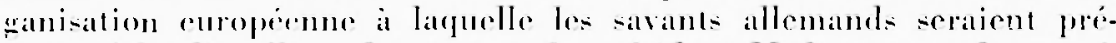
sents. Afiu d'arederer les travaus le presdent Medi propose la consti.

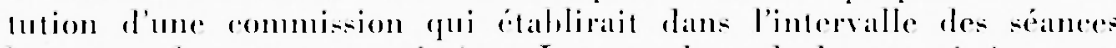
les textes des satuts provisoires. Les memlares de la commission sont chus sur la hase d'un delegue par pays representes all Congres. Sont

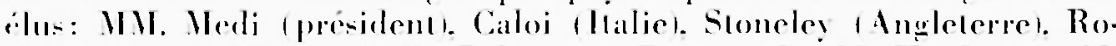

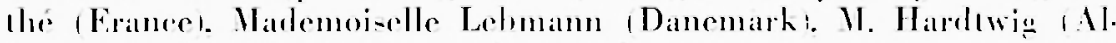
lemannel. Bonelli (E)pagnel, Guyot isuisel.

lal siance est levere à 12 heures 15. 


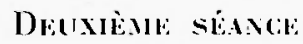

\section{Mercredi 13 septembre (apres-midi).}

La séance est ouverle a 17 heures 30. Le président Medi rend compte des travaux de la commission restreinte. Les idées directrices ont eté celles exposées dans la séance du malin. Le fail dominant est le caractire provisoire des slatuls qui ont été projetes el qui ront être discutés. Ces statuts doivent être ratifés par l'assemblée éénérale de l'U.G.G.l. a Bruxelles. La commission apris longue disenssion s'est en définitil arrête an litre suivant: Federation Eêsmologique Européenne i F.S.E.).

Le lexte des statuts est examiné par l'assemblé générale article par article. De nombreux parlicipants interviennent pour en amender ou en corriser les termes. Le texte définitil figrure en annexe froir paye ... I.

Il est décide que le righlement intérieur sera chabli par le Conscil qui doit être èlu à la fin du Congrios. Ce règlement deva elre ratifie par la prochaine assemblé générale de la F.S.E. I ce propos fuclques idées qénérales sont disculées. Lal eotisation des membres devra être fixée au environ de 10 shilling̣s. Tous les séismologntes de tous: les pays européens doivent pouvoir devenir membres de la Fédération. La convoeation de l'assemblée générale doit etre laile sulfisamment lôt pour que chaque membre puisse laire les demarehes nérensaires. Lat séance est levée à 19 lecures 30 .

\section{TroIsIÈME: SËANCI: \\ Jeudi lte septembre (matin).}

Lal séance est ouverte à 9 heures 15 par Mademoiselle Lelmuma vice-président en l'ahsence du président Medi.

Le Professeur Stoneley présente son élude sur la polarisalion des ondes S. Sur une question du Docteur Grenel. le Professeur Stoneley précise que l'anisotropic choisic de la croûle terrestre a une valeur admissible maximum el qu'il est impossible d'expliquer par ee moyen une différence de temps d'arrive observe de 10 a 14 secondes entre les ondes SH el SV.

Le Professeur Rollé présente le mémoire qu’il a ridige sur la structure de l'Atlantique.

L'auteur rappelle l'bypolbise de travail qu'il arait lormulée en 1947: l'Océan Atlantique est divisé en deux domaines, me zone occidentale à caractère simatique et une parlie orientale à structure continentale. à l'est de la crête médiane.

L'auteur envisage la repatition des loyers seismiques, la forme des côtes, la prolongation en mer des mutes morphologiques afrj-

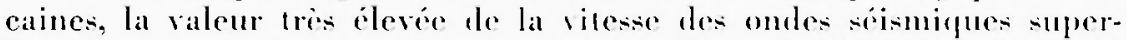
ficielles se propageant sous la partie ocedentale de l'Atlantique. Il discule l'étude de P. Caloi el de ses collaborateurs et apporte de nouveatx arguments en faveur de son hypothese: draguages d'ume 
tachylite ('Temier), de Trilobites (Furon), de calcaires d'âge tertiaire (Ewing et Tolstoy) dans la partie orientale de l'Atlantique; théorie des chanes liminaires (Glangeaurl); resultats des prospections séismidues par refraction obtemus à l'ouest de l'Ecosse fllill el au voisinarge des Bermudes (Ewing).

Te Professeur Calos présente une refutation d'un certain nombre d'arִwuments.

1) Ies ondes par Caloi, Marcelli, Pannocehia indiquées par $L_{4}$ pour la station de Tishonne ne peuvent pas etre conlondues aver SS, SSS. Ieur temps de trajet et, surtout, leur periorle n'arhmettent pas de doules alu rearard.

2) Pour les raisons exposées dans leur deuxième note (Caloi P., Marcelli La, Pannocelia G. - "Ancora sulla velecità delle onde su. perficiali per trag̣tti sulatlantici, ece. Anmali Geofisica, III, 2, 1950 n'est pas consenti d'attriluer les mêmes caractéristiques élastiques aux "redroterra " ("hinterland ) I opposés de l'Mtantique.

Néammoins, à confirmation de la grande vitesse des ondes $L_{\text {q }}$ même dans le hassin oriental de l'Alantique, outre à la station de Lishonne, il y a la limoignage bien plus incontestable de Kew; oì les ondes $l_{\text {a }}$ apparaisent tris nettes el montrent une vitesse encore phus srande ale celle enregristré a Otawa, de $1,3 \mathrm{~km} / \mathrm{sec}$ au moins.

31 I propos de la presumée soudure de la ceinte séismique méditerrancomme aver les lgores, on ne voit pas comment, s'elle existe, peut témoigner de l'equivalenes grologique des régions étendues telles que l'burope, l'Afrique el l'dilantique oriental.

La sínmiciti press des îles Arores est tout à fatit différente de relle re la peninsule llérigue. De plus. La remarguable densité des épientres qu'on observe le long de toute la dorsale centrale atlantique. rompare aver relle triss failite de la fosse entre les Acores et la peninsule Ihérifue, constitue une preuse tres chaire du défaut de sourlure entre les deux zones séismigues de la Merliterrance et de la dorsale atlantique.

l.) Non seulement les deux zones de l'Mtantique ont une batlymétrie tres comparable mais prespue évale est ans-s leur séismicité.

5) Ia dicouverte des matériels sialiques en quelques endroits sur le fond de l'dulantique oriental, pres de la dorsale mérliane, n'est pas

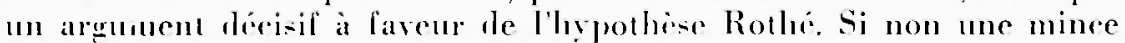
comehe rontinue, au moins des îles sialiques peurent hien exister sur le lond le l'Atlantique. Ene telle courhe, eppendant, si elle existe. doit etre tris mines d'une epaisseur de 40 kms priss en correspondance du sytime alpin, elle devient de $20 \mathrm{kms}$ pour le Wiirtemberge, de $16 \mathrm{kms}$ pres pour la Rhénanie et se reduit a lo kms pres pour les iles luritannirges.

6) Dijà cu 1925 Krenkel avait supposé que à occirlent de l'Afrique les formes du fond océanique deuotent une continuation des lignes fondamentales de l'ossature continentale: mais elte affrmation. eréologiquement indémontrable, n’avat qu'ume signifieation polémique, invoqué eontre la theorie de Vegener. 
7 Le prof. Caloi considie insoulenable la theorie de Wegrener. Dans le cas specifiefue, si la séparation des Ancériques se liul verifiéc le long de la dorsale allanlique, les cnormes lractures ainsi leberm:nées auraient dû donner au côte occidentale de la dorsale un "lalus" (" scarpata ", "Böschumg $)$, avec une pente plus ou moins acenluce: lamdis que a l'orient le lond de l'Océan elevrail etre assez plus relere. La dorsale, au rontraire, justifie en plein son nom el a loutes les caracléristiques al'une montagne submerwee.

Lat morphologie done ne témoigne ni pour la supposie ruplure. ni pour l'éloignenent.

8) La hathymétrie présent des fosses el des planes sulmerocess de comparable profonaleur aux deux cotes de la dorsale. Si nous desonretenir valahle la thérie isostasidue fel telle elle est reteme par les plus comnus géodites el geologuest, spécialenent dans le developpement a elle donne par Pratt. l'épuilibre isostasique serail impossible non seulement entre les deux zones de l'M lantique. mais. hiou plus. entre la zone orientale el les eontinents limilrophes.

Le Professeur Mintrop invoque les lois grencrales le l'isostasie. L'épulibre isostasique est réalisce d'une lacon gémérale sur les gramelcontinents, en Afrique el en Sie en partirulier. Fin appliguant les corrections de Bouguer on aboutit à une anomalie négalive soms les mentagnes et à une anomalie positive de 30 milipals solt les potondeurs oceaniques. La theorie du flotlement des continents sur le magma sous-jacent est un non sens geologigue. Il faut aussi rematrquer anomalie positive de 230 milliwals dan- la ner Thỵrbénienne: en re

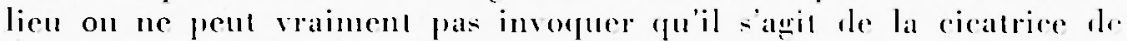
la lume.

Le Professeur Rothé répond que l'anomalie positive de la mer Thỵrhénienne est lice a nue anomalie negative. On a dans a ras une struclure analogue a eelle des ares pacifiques lécrits par Gutene here. Lexistence de tremblements de terre a foyers intermediater confirme ce tait. Il insiste sur l'existence réelle diune zone seismigpe reliant la zone de la erete médiane de l'Allantidue a celle des séismes mésogéens.

Monsieur Pelerschmitl invorpant les travaux de Gulenherer indique qu'il serait possible de dommer une preuve de l'existence d'une surface de separation entre hlocs oceaniques el continentatux dans l'Atantique du Sud. De mene que les ondes superfieielles provenant des seismes japonais d'une part, peruviens l'antre part arrivent a Pasalena fortement amorties far suite de refractions multiples a la surface de separation du bassin pacifique el lu hloe americain. il se pourrat que l'on puisse observer un fait analogue pour les séismes de l'Allantique Sud. Si une surface le separation existe dans celle region, on devait obtenir des valeurs de magnitules trop laibles pour les stations de l'Europe occidentale el normales pour les autres.

La séance est levéc à 11 heures. Pendant l'interruption les congrésistes ont loceasion de visiter sous la direction de Messieurs Zorzi. Conservateur el Paza. Assistant Giologue la belle collection géoloni. 
que du Wusenu d'llisoire Nalurelle et d'admirer en particulier la magnifique carte weologique en reliel elahlie par Monsicur Paza. Les délials sont repris a 1.1 lecures 15.

Ice Doeleur Ilardwiz expose sa note "Lntersuchungen iiler Mikroseismik während der Krieges in Deutschland n. Il insisce en particulier sur le lail qu'il a cli impossihle de rattacher l'agitation microsésmique increvite en fiurope ocedidentale aux lépressions barométriques circulant sur l'Alantique, la mer du Norrl et la Manche.

Mardemoisclle Lehmann lait remanguer que le Docteur Harritwirs

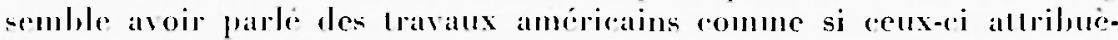
rent uniquement l'agilation meroseismique aux dépressions. Ceci n'est pas le cas. Guilhmore dans sa puldicalion a domni une figure représentant les variations de l'agilation pendant un temps assézétendue. Guelques cats de tempêtes microsédsmiques peurent etre raltachés à

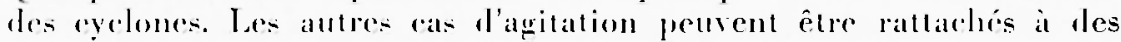
influener diverses, par exemple les fronts froids.

le Docteur llonzel rappelle la lhérie de Press et liwing: dans les fortes depressions siluces au-dessus de la mer, le l'énerqie est transmise sous lorme d'ondes de compression vers les fonds océaniques el donne lieu ainsi a l'anitation microscismique. La période des ondes produiles est calculalile: elle est proportionnelle a la profondeur de lat mer. Ie Docteur Menzel croit que cette théorie permet dexpliquer l’imposibilite dans laquelle on se troure pour rattacher laghtation marosionique européenne aux dépresions. En ellet une dépresion sur l'Alantique engendreratit bien une aqiation de période de 3 a lo recondes. Mais les ondes traversent sur leurs pareours vers l'Furope des aceident- géologidues prolonts où elles doivent perdere leur enereie anos que l'a prouve Gutenlerer. Par contre une depresion situce sur la mer du Sord engendremait des ondes de pé. riorle tris faible infrerienre a 0.1 seconte. Op les ondes de si faihle pérode sont fortement alsorlues el les séismographes des stations curopreanes ne sont en genéral pas adaples a leur inseription.

Le Docteur Harlowig fait remarquer que d'apros les travaux américain- la predison de la delermination du rentre des depressions par l'emploi de sations tripartiles est home senlement quand il s'agit

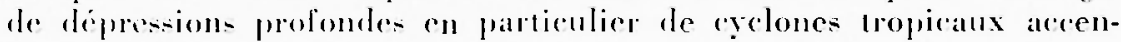
intis.

I. Prot. Caloi. termine l'exposition du Dolt. Harltwiz fail quelfues obervations. Dis 1936 il a prouse la weneration de microséisnes m rorespombance de la phase positive de la pression. relative aut pasage de noyaus rgeloniques le long̨r de la haule Arlpiatique. Ine conclusion analegue ont trouse tout reemment les proll. Gioreri rt Rosmi. pour a qui concerne la Thymhine. Sont en rours des re.

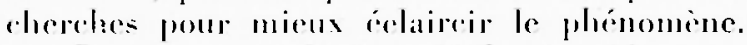

la siance al levere a lo lecures 15. 


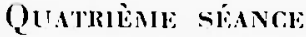 \\ Jeudi 14 septembre (apres-midi).}

La siance est ouverte a 17 lieures 30 .

Le Docteur Grenet lait un exposi sur les raracteristipues des seismographes clectromagnéliques. Parmi les sëismographes modernes on peut distinguer:

a) l'appareil Bénioft courte période dont les aaractiristipues sont: période propre du pendule $p=0.5$ sec. periode propre du walvanonelre $g=0.2$ sec, grandissement maximum $\mathrm{V} m=100.000$. C.el appareil présente l'inconvénient d'amplifier fortenent les trés courtes périorles et d'être ainsi sensible aux perturlations locales.

b) les appareils courte période du upe Grenet. Willmore, Pe. tersehmilt. On a dans ee cas $p=g=1$ sec. $/ m=5000$. Ce lype d'appareil est moins sensihle aux perturhations locales dans la hranche descendante de la courbe de prandissements, il est a peu pres identique an Béniofl.

c) Le lype Bénioff gramle période $p=0,5 \mathrm{sec} . g=90 \mathrm{sec} \cdot$, donne des résultats analogues au lype Grenet $p=y=10$ sec. l'amortissement étant éal a 7 fois la valeur critipue. Ce ype est caractériós par une amplifieation quasiconstante dans un domaine ctendu de periodes. 11 faut remarquer une prédominane des ondes à counde pirionle.

d) un dernier type l'appareil est le montage Faux Venner Grenel $p=10$ sec., anortissement 10 fois la valeur erilipue, $g=-60$ sec., anortissenent critipue $/ m=200$. Ce montąge se distingue du préérlent par tue amplification moins prande des ondes a courte période.

Le choix des stations a une imporlance tres elande. Le Docleur Grenet cite le cas de la station de Tamanrasset où maleri la forle amplifieation des séismographes aus périodes noyennes l'apitation microséisminge n'apparait pas.

L'Inquénieur Gomez-Guillamon fait une description d'un montawe réalisé a la station de Malaga. Un seismographe Victoria a clé construit suivant le morlele du Bénioff. Les caracteristiques en sont $p=0.3$ sere. $g=7$ sec. Dautre part un vertical Wiechert $80 \mathrm{~kg}$ a ife modifis en séismographe du type Galitzine. La periode propre du pemdul. est de 12 sec. Le Victoria el le Wiechert sont hranche sur lo meme galvanemètre: on ahoutit ainsi at une rourle de grandisiement pré. sentant denx maxima $(\mathrm{V} m=1.500)$ ca.l. l'un au cmiron de 0.3 sec., l'autre vers \& sec. L'Ingéineur Gomez-Guillamon présente quelefues photographies ales appareils el puelpues cureqistrements.

Wonsieur Pelerschunitl insiste sur le choix de l'emplacement des stations. Une comparaison cutre les inscriplions des verticaux conte période de Stutgart et de Strasbourge a montre que les deux alpatreils sont sensihlement éfuivalents. Les enregistrements de Stultgart sont meilleurs avant tout par l'alsence d'agitalion microséismigue industrielle. Il rappelle les travaux du Professeur Lateoste: l'apitation 
méléorologique est 2,5 lois plus faible à Sainte-Marie-aux-Mines, ville siluce sur le qranit des Vosges, qu’a Strashourg dans le fossé rhénan.

Marlemoiselle I.ehmamn a remarqué que de même qu'à Strasshourw, un grand nombre des enregistrements de Stullgart sont à Copenlague masques par l'agilation industrielle.

Le Docteur Grenel signale gue l'on pourrait songer à placer des séismographes dans des puits de mines. I'éplange de vue auquelle participent en particulier MM. Mintrop, Menzel, Schulze et Peterschmitt montre qu'il ne faut pas a attendre de grands résultats de cette lacen de procéder. L'agitation métcorologirque n'est pratiquement pas diminuce el d'autres perturbations locales apparaissent.

M.P.L. Willmore Tait une deseription detaillée du séismographe electromagnétique transporlable de sa construction. Ial description est illustric par la presentation de son appareil en ordre de marche.

En l'absence du Prolessour Mihailovic, le Professeur Rothé pré. sente le nouveau reglement du Service Séismologique en Yougoslavie.

Ie Professeur P. Caloi résume rapidement la mémoire "Sulla superficie di discontinuita alla profondita di $950 \mathrm{~km}$ (a. ").

Ahordant le dernier point de l'ordre du jour Mademoiselle Lelımann donne les résulats des travaux de la commission restreinte qui avait siege avant l'assemblée générale en rue de proposer une liste de membres du Conscil de la Fédéralion. Aprés un échange de vue l'asemblic passe aux clections qui ont en lien a lubletin secret. i l'exception de celle du Secreatare General qui esl cilu par acelamation. les reultate des votes sont les suivants:

-.- Presirlent: Marlemoisclle Ichmamn:

- Vice-Prosident: Monsicur Pelerschmit:

- Secretaire Gincral: Monsieur Caloi:

-_onseillers: Monsieur Bonelli, Monsieur Hiller.

l.a siance est leve a lo leures 45.

\section{JoLRSLE: DU 15 SEPTEMBRE \\ Fixarsion geologique au Lac de Garde.}

Entre firone et Pescliera les coneresistes traversent l'armiratble amphitifatre morainique qui s'itend au Surl du Lare de Garde, puis montent de lone du liord oriental du Lar juequ'à Riva. Une halte ; la pointe de San Viuglio permet de voir en place de Batlonien-Bajocien ì prande ammonite et solithe. L'ensemhle jurassidfue forme dans celte région le flane oceidental de l'anticlinal du Monte Baldo. The autre halte a Asonza permet l'aperevoir l'île de Trimelone ou aflenre le Crétaces dans l'axe du syonelinal du Late de Garde. Ies plis de part el d'autre retombent presque vertiealement. A l'entré de Malcesine les congrésistes examinent tun tuf hasaltique petit limoin des vastes ermption qui phts a l'Est ont dome nassance à d'immenses couleses. I l'entréc de Riva dans laxe de la vallée au- 
jourel'hui oceupe par le Late s'iblivent les collines miocines du Monte Brione dome le pendage leaweoup plus doux rombrase avee les plis jurassigues redressés.

Le dejenter pris en eommun a Riva donne l'ovasion aux congrésistes de remereier les organisaleurs du Congres de leurs ellorts. Fin partieulier Matemoiselle Lehmam, Messienrs Baxler, Rollá, Bonelli, Mintrop expriment leur profonde recombissanee el félieitent leurs ollewues ilaliens de leur si parfaile orquatusation.

Apres le dejenter à Riva les congressistes visitent les rascades somberatues de Varone. Ces caseades oecupent un poulel ereuse à l'interieur d'une masse de ealeaire tres compare du jurasigue supe-

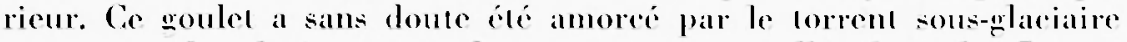
provenant du ghacier suspendu oceupant une vallece laterale. Le recour śeflecelue par la rôle occidentale du hate en suivant une rente presque ronstamment lailléc en lumnel. Fintre Camprone el Garynano. la route traverse l'anliedelinal jurassique du Mont Denervo sur les lords duquel vient en reourrement anormal la dolomie triasique.

A 20 heures 30 un hanquer olfert par lo Conseil Municipal de Verone rémit pour la demieme fois les membres fondaleurs de la Fedération Seismologique Europécome. Mademoiselle Lelmann. les Professeurs Rothe el Caloi en profitent pour remereier le Gourernement halien el la ville de Verone pour sa chaleurense hospitalite. Monsienr le Maire dans sa reponse lélieite les compressintes daroir travaille dans un si bel areord. 


\title{
FEDERATION SEISMOLOGIQUE EUROPEENNE
}

\author{
$\mathrm{S} \mathrm{T} \Lambda \mathrm{T} \mathrm{U} \mathrm{T} \mathrm{S}$ \\ adoptés à la riunion de Verone (Septembre 1950).
}

I. - Ie bul de la Federation Séinmologique Luropéenne (F.S.E.) est de permettre une plus grande collalioration entre les séismologues européens par des rencontres fréquentes pour l'étude en commun de problemes particuliers.

II. - Les staluts cielessous sont provisoires el devront être ratiffés par l'assemblée générale a Bruxelles.

III. - La F.S.F. est placéc sous le patronage de l'Association Internationale rle Séismologie.

IV. - Toute institution ou personne t!availlant dans le domaine dfe la séismologie ou dans des domaines connexes peut devenir memlere de la Federation.

V. - I.es ressources funancieres de la Fédération sont assurées par les cotisations des membres. par des subventions diverses et par des dons.

VI. - Le Conseil et l'Asscmbléc générale sont les organismes de la lederation. Le Conseil de la Federation romprend le Président. Ie Viec.l'résident. le Secrétaire Général et deux Conseillers. Les membres du Conseil, outre le Secrétaire Générale, restent en fonction pendant deux periodes administratives: la periode administrative est l'intervalle de temps qui s'écoule entre deux assemblées énérales consécutives.

VII. - Le renouvellement par moitie des membres du Conseil à l'exception du Secretaire Géneral a lieu a la fin de chaque assemblé générale. Sauf pour le Secrebaire Général, les membes du Conseil ne peuvent pas etre rélus immediatement. Le Secretaire Général est elu pour trois periodes arministratives consécutives et est réligible.

VIII. - Le Conseil examine et se pononee sur des demandes d'admission des nouvalux membres. Les admissions doivent être ratifices par l'assemblé énérale stivante. La radiation d'un memlere peut etre prononcé conformément ax dispositions prevues par le réplement.

IX. - Ies as-embles générales de la F.S.E. doirent en principe. coincider avee los assemblees générales de l’T.G.G.I. En outre au moius une antre asembléc doit être tenue dans l'intervalle separant delix assembles générales de l'T.G.G.I.

X. - Toules les décisions sont prises à la majorité simple des rotes exprimé laver l'exeeption mentionné en XI. Le vole par délexation est autorise a condition que la dékexation soit donné par ceril pour chacune des questions qui figurent à l'ordre du, jour. En memlure present ne peut pas être porteur de 
plus de quatre delexgations. Auenne decision antre que eelles concernant les questions fiqurant a l'ordec du jour ne peut etre prise.

XI. - Tonte proposition de modifitation atux presents statuts devat être transmise at Conseil al moins trois mois avant l'assemblec erenerale el le Conseil devra en informer les membres au moins un mois arant l'assemblé générale: la majorite des deux tiers (2/3) des voix de membres votants est requise pour l'approbation d'une modification aux statuts. 


\title{
FEDERATION SEISMOLOGIQUE EUROPEENNE
}

\author{
S T A T U T O
}

I. - Scopo della Federazione Sismologrica Europea: permetlere una più intensa collaborazione fra $\mathrm{i}$ sismolori curopei, con frequenti contatti per lo studio in comune di particolari problemi.

II. - Lo statulo che segue i provisorio e dovà essere ratifieato dall'Asiemblea di Bruxelles.

III. - La F.S.li. i posta sotto il patronato dell'.S.A.

IV. - Posiono far parte della Federazione le istituzioni e ie persone fisiche. rhe lavorano nel campo della sismoloria o in campi connessi.

V. - I mezzi linanziari necessari al funzionamento della Federazione sono assicurali dalle quote sociali, da sorvenzioni e contributi vari.

VI. - II Consirlio e l'Asemblea Generale sono trli oromi della Faderazione.

11 Consiglio a costituito dal Presidente, dal Seqretario Generale, dal Vice-Presidente e da due Consigrlieri.

I membri del Consiplio, olte al Serretario, restano in carica duranle due periodi amministrativi; un periodo amministralivo va fra due Assemblec Generali.

VIl. - Il rimnoro per meta dei membri del Consirlio, latla eccezione del Sepretario, i elletluato alla fine di orni Assemblea Generale.

Ad eccezione del Sewretario, i memliri del Consiglio non gossono essere rieletti immediatamente.

Il Segretario is eletto per lre periodi amministrativi conseculivi al $a$ rielegribile.

IX. - Le Asemblee Generali dovamno di norma coincidere con lo Assemlilee Generali dell'T.G.G.l.

Inoltre. un' Asecmblea almeno dere aver luogo nell'intervallo lira due assemblee dell't.G.G.l.

X. - Tutle lo decisioni sono prese a semplice magrgioranza dei roti espresi ceon lecerzione riportata in XI. Sono ammessi i roli per delegar a condizione che la delega sia data per questioni che freurano allordine del riorno. Un membro non puo porlare piu di fuattro deleghhe. Nessuma decisione, al di fuori delle questioni previste allordine del giorno. può essere presal.

XI. - Ogni modifica al presente statuto deve essere proposta al Consiolio almeno tre mesi prima dell'Asemblea Generale e dal Consirtio stesso commintata ai soci almeno un mese prima dell'Assemblea Generale. La modifica richiede per l'approvazione i due terzi (2 3) dei roti espressi. 


\section{FEDERTTOX SELSOLOGIQTE EUROPEENNE S T}

I. - The aim of the Féleration Söismologicue Europienne (F.S.le. is the allainement of eloser collahoration hetween European Seismologists through frequent mectings for a common sturly of particular prohlems.

II. - The statutes are provisional and should he suhmilled to llar General Asomhly at Bruxelles for ratifieation.

III. - The F.S.E. is under the patronage of the I.S.A.

IV. - Institutions and persons working in scismology or in ficlel. closely eonnected with it maty hecone memhers of the Fede. ration.

V. - The income of the Federation is derivel from memhers' sulh. scriptions, from diverse contrihutions and donations.

VI. - The Council and the General Asembly are the Organs of the Federation. The Conneil of the Federation consists of the President, the General Secretary, the Vice-Presilent and ? Councillors. The memhers of Council, other than the General Secretary. holds offec for 2 arlministrative terms. The arlminstrative term is the time helween 2 General Asemblies.

VII. - Elections will he held al the cond of each General Isembly for half of the members of Councile will the execption of the General Seeretary. Exeept for the General Secretary, members of Council can not he reclected to serve immetiatels after their period of offece has expiret. The General Secretary is elected for 3 conseculive terme and he mas he reclected.

VIII. - The Council examines and reports on applicalions for memhership.

Elections should he suhmilted to the next General Asiembly for eonfirmation. Members may he expelled aecording to the. rules fixed in the hy-laws.

IX. - A General Assemhly shonlt normally coitride with the General Isembly of the I.G.C.I. Al leasi one other Assemhly shall be held in the interval helween 2 dsisemblies of the U.C.G.l.

X. - A simple majority is decisive in all questions a with lle exception mentioned in XI. Voting hy prosy is allowerl. A memher may not represent hy proxy more than I other memhers No decision can he taken on a fuestion that has not heen announced in the programme of the Mecting.

XI. - Any proposal to alter hiese statutes must he sent to the Council at least 3 months hefore the General Asombly and the Council shall inform memhers at leas one month hefore the General Assemhly.

A majority of wro thirels 233 of the votes given is refpuirest for lhe approval of any alteration of the statutes. 


\section{FEDERATION SEISMOLOGIQUE GUROPELNNE $S$ A T Z U N \& E N}

I. - Der Zweck Jer F.S.E. ist eine inlensivere Zusammenarloil unler den europaitiselien Seismologen sowie die wemeinsame Bearlicilung lostinmter Probleme aul haufiæen Zusammenkiinlien.

11. - Die folgenten Satzumgen hahen vorläufiecen Chamaker und sollen von ler Tagung in Brïssel genelunigt werden.

111. - Die F.S.E. wird muler das Patronat der I.S.S. westellt.

IV. - Der Föderation kïnnen Anstalten unt physische Personen an̈rehären, die anl' dem Geliete der Seimolowie orler at dannit zusammenhängenden Gehieten arheiten.

V. Die zur Tatiykeit der Föderation erforderlichen funaziellen

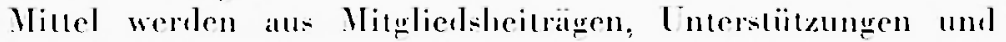
sonstigen Zuwendungen aufyehraeht.

VI. - Die Orande der Föderation -ime der Vorsand und die Generalverwammlung.

Der Vorstant wind rom Präsidenten, rom Generalsekrelar, rom Vicepräistenten unt zwei Beirälen gehilılel.

Dic Mitulieder des Vorstandes hehalten - mil Ausnahme des Generalsekretars -...- ihren dultraw zwei Verwalumgsperioden lanw - eine Verwaltunwsperiole ist die Zeit zwisehen zwei Generalversammlument.

VII. - An Sehluse jeder Gencralversammlung werten die Milwlierler des Vortandes zur llälle emeuert, alu-qenommen der Se. kretïr.

Die Vitglieder des Vorstantes diirlen nicht solort wiedergewällt werten. mit Austalme des Sekretïrs.

Der Sekretar wirl fïr drei anfeinamberfolyente Periotlen wewihlt und ist wieder wählhar.

VIII. - Der Vorsand prifl die Aulnahmeantäge non Bewerhern um die Mitgliedschatt umd äuseret sich darïher.

Die Aulualmen miissen ron der nälisten Generalversammlung enenehmigl werlen.

Gemäss der auf den Satzungen hasierenten Vorschriften können Mitglierler atuseselilosen werten.

IX. - Die Generalversammlunuen sollen wewöhnlich mil den Generalversammlungen der U.G.G.I. zusammenlallen. Mindestens eine Versammlung soll lerner in der Zsischenzeit zwischen zwei Verammlungen der U.G.G.I. slattfinden.

X. - Alle besehliisse werden mit Stimmenmehrheit gefasst - mit Ausnahme des in Xl. vorweschenen Falles.

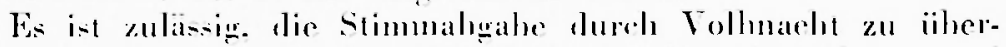
traugen unter ler Bedingung, lats die Yollmachl sich nur aut 
Fragen bezicht, die auf der Tagesordnung steben. Ein Mitglied darf nur vierfach bevolmähtigt sein. Uher Fragen, dic nicht auf ler Tagesordnung stehen, diirfen keine Beschliisse gefasst werden.

XI. - Jede Änderung dieser Satzungen nuss dem Vorstand mindestens drei Monate vor der Generalversammlung und vom Vorstand sellsst den Mitgliedern mindestens cinen Monat vor der Generalversammlung vorgescblagen werden, Der Abänderungsvorschlag erfordert zu seiner Annahme zweidrittel $(2 / 3)$ der aljgegebenen Stimmen. 


\section{AIDESIONI AL CONVEGNO DI VERONA}

Lat Sig.na I. Lehmamn hat accemuato (pag. 579) ai motivi rhe hanno indotto alruni sismologi europei a proporre la costituzione di un'associazione fra quanti si occupano altivamente di Sismologia.

Qui io desidero riassumere le ultime fasi che condussero al Convegno di Verona. In una lettera personale del 16 gennaio di quest anno, la Sig.na Lehmann mi chiedeva se era possibile organizare, al fine su detto, una riunione in unil citta dell'Italia settentrionale. L'Istituto Nazionale di Geofisica aderi volentieri alla proposta.

Fu allora pregato il Prof. Rothe, Direttore del Bureau Central dell'A.S.I., di diffondere una rircolare in proposito. L’invito fu cortesemente arceltato. $\Lambda \mathrm{i}$ primi di aprile, venne infatti diffusa la seguente circolare:

"Le Professeur Pietro Caloi prie le Secrétariat du Bureau Central de diffuser li nole suivante:

Donnant suite à une suggestion de Mademoiselle Lehmann (suggestion approuvée par le Dr. Stonefey, le l'rolesseur Rothé et de nombreux autres séismologues europiens), listituto Nazionale di Geofisira (Romal a acrepte dorganiser une réunion pour lat constitution de l'Organisation Séismologique Européenne (O.S.E.). L'Istituto Nazionale di Geofisica propose que rette réunion ait lieu dans une ville de Jtalie la Xord pendant la seconde décade de septembre 1950 (la date exacte devant etre précisece ultirieurement).

Afin de pouvoir prendre les di-positions nécessatres pour Torganisation de cotte réunion il est desiderable de comnatre le plus tôt possible le nombre approximatif des participant.s. Ceux de no: Collegues européens qui ont l'intention de participer a colte réunion sont priés den avertir le plus lit possible le Professeur P. Caloi, Istituto Nazionale di Geofisicil. Citta Universitaria. Roma (Italia).

\section{Le Directeur du Bureau Central Stratbourg. Ie 28 Mars 1950 m.}

Numerosi sismologi europei e-presero il proposito di partecipare alla progetlata riunione o comundue manifestarono solidarieti all'iniziativa. Essi furono:

Charlier per il Belgio;

Zattopek per la Cecoslovalchia;

Niss Lehmann per la Danimarca:

Eijo Vesanen per la Finlandia;

Debrach, Grenet, l'eterschmitt. Rotla per la Frimcia;

Hardtwig, Hiller, Krumbarh. Menzel. Mintrop. Schulae per la Germania:

Baxter, Browne, Pollard. Stoneley. Nillmore per l'Inghilterra;

Milaialovie, Mokrovic of Vukojicie per la Jugoslavia;

Veldkimp per l'Olanda;

Bobr Modrak per la Polonia;

Bonelli, Gomez-Guillamon per la Spagna;

Bath per la Svezia;

Guyol, Mercier, Oulianoff per la Svizzera:

i proft. Aliverti (l'avia), Aquilina (Roma), Imbo (Napoli), Morelli (Trieste), Solaini (Milino), Vercelli (Trieste), nonche i geofisici dell'Istituto Nazionale di Geo. fisica per l'Italia. 
Per un complesso di motivi, indipendenti dallit loro volonti, alcuni dei sismologi sopra citali non poterono prender parte al Consegno, per il qualle, nel frattempo, eral statia sceltal come sede lat citti di Verontal

Prima del Convegno, veme distribuilo, a coloro rhe avevano assirurallo il loro

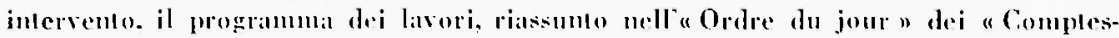
Rendus " (piar. 581 ).

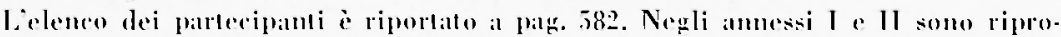
dolli i progetti di statulo, claboralti il primo dalla Signal Leluminn a dal Prof. stoneley e il secondo dall'Tstituto Nazionale di Geofisiral.

Talli progetti fornirono la bitse fer leclaborazione, in comme, dello statuto

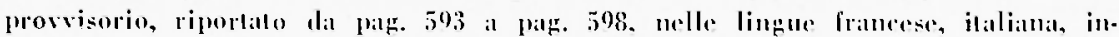
glese e tedesci.

P. Calor

Dicembre 1950 


\section{ANNEX: I \\ THE ELROPLAN SEISMOLOGOAL ORGANISATION ORGANISATION SEISWOLOGIQUE EUROPEENNE ,O.S.F.।}

Propusal of hy-laws

(IForliet out by I)r. Sioneley and I. Lehmami)

The object of the Organisation is to encourage the science of seismology in Europe by affording opportunities for seismologists 10 meet and discuss seimolo. piral prohlens and to confer on topies relevant to the development of their science. Meetings will ordinarily he helt at intervals of alhout eighteen months.

Membership of the Organisation is open to scientists working in seismology or rlosely related fields of study.

The officers shall he dhosen from among the memhers of the Organization and shall consist of a President and a Ceneral Secretary. These officers with three other members of the Organisation shall ronstitute a Council for the management and direction of the affairs of the Organisation. The Council may, at its discretion. desiEnate one of its members als lice-President.

The Presilent, the General secretary and the thee other members of the Conncil slatl he elected by ballot at the li/2 - yearly meeting. The General Secrotary may he recelected so as to serve for three consecutive periods of office. No other member of the Council may serve for more than two consecutive periods. Of the four members other than the Ceneral secretary not more than two may be re. elected at the same time. After the expiration of one period in which he is not loolding office a former member of Council may he elected anew.

Any member all bave power to nominate any other member for election as Presdent. Ceneral secretary or member of Council. Nominations mus he received by the General secretary not lese than two months before the opening day of the Meeting. I list of the names of the members nominated for the various office: stall he semt to all members not later than 3 weeks hefore the opening day of the Meeting. Hembers not present at the Meeting may send their votes hy post, making use of the balloting lis.

The Council remains in offece until the Meeting following that of it election if it is not found posible to hold this Meeting after the ordinary interval of time.

Ipplication. for membership must lore sent to the General Secretary and must bear signature of two members of the Orgatnisation.

Members are elected by the council. but the elections are submitted to the mext Yeeting for confirmition. A tis of the members elected shall he di-tributed in advance of the Merting. If an ohjection is raised anginst any person elected the eandiclature shafl he dierusecel and a ballot taken.

Notice of a motion for the expulsion of a member must he received by the Ceneral secretary not later than 28 digs before a Heeting and must he circulated to members before the Meeting. I oting shall be by ballot. and for the expulsion of a member a $2 / 3$ majority of the menbers present shall be required.

The subscription shall he hept as low at possible. The Council shall submit to each lleeting a profusial for the amount of subscription payable for the following $1 \mathrm{l} / 2$ yearly period. A tatement of the subseription due shall be sent to eath member ats soon after the Heeting ats practicable, and subscriptions shall be posted 1. the General secretary within two months of the date of the satement.

If, after due notice, the substiption remains unpaid. the Council may, al its 
discretion, declanc that the menher has forteited his membership of the Orgatnisation.

The Council shall organise the Mreting of the Organisation. The place and date of the Meeting shall be announced at as eatly a date als possible, and not later than three montlis in advance.

Members desiting to read papers or to open discussions at the Mceting must send in titles and short summaries not later than lwo months before the Meeting. Members desiring to hatve questions relative to the affairs of the Organisation discussed at the Heeting should also send their proposals to the Council two months in advance.

A programme of the proceedings shill be circulated not later than three weeks in advance of the Meeting.

The Meeting will receive the Comeil's report on the activity of the Organisation and on the Findncial status. The subscription for the coming $1 \frac{1}{2}$ - yearly period will he decided on. Election of officers and the confirmation of the election of members will take plate. Questions relative to the affairs of the Organisation may be discussed and determined, but no decision can be taken on a question that has not been raised beforehand and amnounced in the programme of the Meeting. A simple majority is decisive in all questions except the expulsion of a member and any alteration of the by-laws, for which a majority of $\frac{33}{3}$ of the votes given is required.

In accordance with the object of the Oryanisation the hringing formard of seismological problems and discussions tendine to promote seismology shall constitute the main aletivity of the Heeting. The Council decides on the duration of the Heeting which may vary alecording to the numler and importance of the topies 10 be consired.

The Council shall have the direction and management of the affitirs of the Organisation subject to the control of the Meetings of the Organisation.

The Council shall draw up a report on the state of aftairs of the Organisation to be presented it the $11 / 2$ - vearly Meeting.

The duty of the President shall be to take the chair at the Heetings of the Organisation and to regulale and keep order in their proceedings; and to carry into effect the by-laws of the Organisation. In the absence of the President one of the members of the Council shall carry out his duties.

The President or any other momber acting as President shall not vote on any question bronght before the Meeting unless a casting vote is required to decide the equestion.

The duty of the General Siecretary shill be to allend the Meetings of the Organisation, to take minutes of the proceedings and to cause them to be entered into the book provided for that purpose.

The General Secretary shall have the manigement of the correspondence of the Organisation, subject, however. to the direction and rontrol of the Councit.

The General Secretary shall also be the treasurer of the Organisation. He slatl heep a regular account of receipts and payments and rive a receiplt for every contribution received. The Council has the direction and control of the money in the lands of the General Secretary.

The accounts of the General secretaly shatl be andited at each Heeting by two anditors chosen by the members.

Derember 19.19 


\title{
ANNExe II
}

\section{SCIIEMA DI STATUTO}

\author{
Unione Sismologica Europea \\ (Serione I.S.A.) \\ (Proposto dall Istituto Nazionale di Geofisica)
}

Scopo dell't.S.E. è il conseguimento di una maggiore collaborazione tra i cul. tori di Sismologia, promovendo e favorendo iniziative dirette and incrementare ogni forma di rapporto scientifico fra i Sismologi Europei.

1. - LU.S.F. ¿̀ la Sezione Turopea dell'I.S.A.

II. - Possono divenire membri dell'f.S.E. gli scienziati che lavorano in sismologia o in campi di studio strettamente comnessi, secondo le modaliti stabilite dal Regolamento.

Hamno diritto ad appartenere all'U.S.E. i soci dell'I.S.A.

III. - I mezzi funanziari necessari al funzionamento dell'U.S.E. sono costituiti dalle quote sociali, da sovvenaioni e contributi vari.

IV. - I'Y Y.S.E. lai sede nel luogo di residenza del Segreturio.

V. - Il Consiglio di Presidenzal d costituito dal Presidente, dal Vice-Presidente, dal Segretario Generale: e da due Consiglieri.

VI. - Il Consiglio resta in carica tre amni.

I Membri non possono essere eletti più di tre volte consecutive.

VII. - I Membri sono eletti dal Consiglio, secondo le norme stalbilite dal Rego. lamento, ma le elezioni sono sottomesse all'Assemblea nella successiva riunione, per la confermit.

I Membri possono essere deposti serondo le modalitia stabilite nel Regolalmento stesso.

VIII. - Il Consiglio di Presidenza si riunisce almeno ogni sei mesi.

L'Assemblea Generale viene convocatit dal Consiglio almeno unil rolta l'anno.

IX. - Tutte le decisioni verramno prese al semplice maggioranza. I membri del Consiglio rengono eletti nell'Asemblea Generale. Sono consentite le deleghe secondo le modilitit stabilite dal Regolamento.

X. Ogni modifica al presente Statuto deve essere proposta al Consiglio di Presidenzil almeno tre mesi primil dell'Assembleal Generale e dal Consiglio stesso. comunicatia ai soci almeno un mese prima dell'Assemblea Generale. Ial modifica richiede per lapprovazione i 23 dei voli dei soci.

Agosto 1950

Diretlore: Prof. ENRICO MIEDI

Prof. Pierro Caroi - Responsabile

Istituto Grafico Tiberino - Via Gaetil. 14 - Romal (Offcine Graffiche, Tiroli) 